\title{
Dynamical many-body corrections to the residual resistivity of metals
}

\author{
V. U. Nazarov, ${ }^{1}$ G. Vignale, ${ }^{2}$ and Y.-C. Chang ${ }^{1}$ \\ ${ }^{1}$ Research Center for Applied Sciences, Academia Sinica, Taipei 11529, Taiwan \\ ${ }^{2}$ Department of Physics and Astronomy, University of Missouri, Columbia, Missouri 65211, USA
}

(Received 4 March 2014; published 30 June 2014)

\begin{abstract}
The residual resistivity of metals at the absolute zero of temperature is usually understood in terms of electrons scattering from random impurities. This mechanism, however, does not take into account dynamical many-body effects, which cannot be described in terms of a static electron-impurity potential. Here we show that dynamical corrections to the resistivity, already known to play a role in nanoscale conductors, are of quantitative importance in the calculation of the residual resistivity of simple metals, and lead to a significantly improved agreement between theory and experiment in the case of impurities embedded in an Al host. Our calculations are based on a recently proposed form of the time-dependent many-body exchange-correlation potential, which is derived from the time-dependent current density functional theory. Surprisingly, we find that the largest correction to the residual resistivity arises from the real part of the exchange-correlation kernel of time-dependent current density functional theory, rather than from its imaginary part. This unexpected result is shown to be consistent with recent theories of the dynamical corrections to the resistivity of nanoscale conductors.
\end{abstract}

The calculation of the electrical resistance of conductors is one of the basic problems in the theory of electronic transport [1]. At the absolute zero of temperature the crystal lattice becomes irrelevant (no phonons) and the origin of the resistance is traced to electron-impurity and electron-electron scattering. Electron-electron scattering conserves momentum, so it is customary to assume that electron-impurity scattering plays by far the dominant role. This is the basis for the standard theory of residual resistivity in normal metals in the diffusive regime. Also in coherent nanoscale conductors, where the diffusive model is no longer applicable, the resistance can be expressed in terms of the transmission probability of noninteracting electrons from one lead to another according to the Landauer-Büttiker formula [2-5]. Electron-electron interaction corrections to the resistivity are known to exist and can be included in principle by various methods, such as the nonequilibrium Green's function formalism [6-8], leading to the Meir-Wingreen formula [9] for nanoscale conductors, and more recently, the time-dependent current density functional theory $[10,11]$. Part of the correction is, in a sense, trivial—at least from the conceptual standpoint. This is the correction that arises from the many-body "dressing" of the static effective potential in which the electrons move. The dressing in question is essentially a generalization of the classical screening idea to include so-called exchange-correlation (xc) effects in the local environment of an electron $[12,13]$. If standard density functional theory $[14,15]$ is used to model these xc effects, great care must be exerted, in nanoscale systems, to ensure that the effective potential has the appropriate discontinuities as a function of particle number [16,17]. This is because the number of electrons in a nanoscale conductor typically fluctuates between $N$ and $N+1$ during the conduction process. Besides the many-body dressing of the static effective potential (which exhibits the discontinuity as a function of $N$ ), there is an additional effect arising from the fact that the conduction process is a nonequilibrium one and therefore cannot be rigorously described in terms of a static effective potential: What is needed is a dynamical effective potential, such as the one introduced in time-dependent density functional theory
(TDDFT) [11,18]. Corrections to the resistance, which arise from the dynamical character of the effective dressed potential, is what we mean when we talk of "dynamical many-body corrections." The existence of dynamical corrections to the resistivity was first implicitly recognized in the nonequilibrium Green's function formalism of Meir and Wingreen [9], but the correction was believed to vanish at zero temperature. It was only in later work, based on time-dependent current density functional theory (TDCDFT) $[19,20]$, that the dynamical corrections were explicitly formulated in terms of a dynamical $x c$ potential and were shown to survive in the limit of zero temperature [21-26]. In these papers the additional dynamical resistance of a conductor was interpreted as a manifestation of the many-body electronic viscosity $[27,28]$ affecting the inhomogeneous flow of electrons in a nanoconstriction or around an impurity.

Unfortunately, the complex behavior of the many-body viscosity as a function of frequency and temperature in an inhomogeneous electron gas, combined with ambiguities in the characterization of experimental devices, has so far frustrated attempts to reach definite conclusions concerning the importance of dynamical corrections in concrete situations [22-24,29,30]. Very recently, however, an interesting paper by Kurth and Stefanucci [31] has conclusively shown the importance of dynamical corrections in an Anderson impurity model in the Coulomb blockade regime.

In this Rapid Communication we consider the somewhat simpler problem of the residual resistivity of a normal metal (Al) with embedded impurities. The application of density functional theory to this system is not plagued by discontinuities in the xc potential and we can thus focus on dynamical corrections in their pure form. We apply the method introduced in Refs. [32,33] to extract the dynamical effective potential of time-dependent DFT from the xc kernel of time-dependent current DFT. In those papers, large many-body corrections to the stopping power of an electron gas for charged impurities were found. The outcome of the present study is twofold: On the one hand, we show that the dynamical corrections to the resistivity for this classic problem are indeed very significant 
and must be taken into account if a quantitative comparison with experiments is desired; on the other hand, we discover that the dominant contribution to the dynamical correction is quite different from the one that was proposed in earlier papers on the basis of a perturbative analysis of time-dependent CDFT: It does not come from the imaginary part but from the real part of the xc kernel, and from this point of view, is more consistent with the treatments of Refs. [30,31].

We start by deriving an exact formula for the frequencydependent resistivity in terms of quantities that can be calculated entirely within density functional theory. Let us consider a monochromatic and uniform external electric field $\mathbf{E}_{\text {ext }}(t)=\mathbf{E}_{\text {ext }} e^{-i \omega t}$ applied to electron gas with impurities positioned at $\mathbf{R}_{k}, k=1,2, \ldots$ We can write the (particle) current density averaged over the normalization volume $V$ as [34] (we use atomic units, i.e., the electron charge and mass are set to unity unless otherwise indicated)

$$
j_{i}(\omega)=\frac{c}{i \omega V} \int_{V} d \mathbf{r} d \mathbf{r}^{\prime} \hat{\chi}_{i j}\left(\mathbf{r}, \mathbf{r}^{\prime}, \omega\right) E_{\mathrm{ext}, j},
$$

where $\hat{\chi}_{i j}\left(\mathbf{r}, \mathbf{r}^{\prime}, \omega\right)$ is the current-current response function of the inhomogeneous electron gas with impurities. A summation over the repeated Cartesian index $j$ is implied. (Notice that the electric charge current is the negative of the particle current.) We transform Eq. (1) with the help of the sum rule [35]

$$
\begin{aligned}
& \left(\omega^{2}-\omega_{p}^{2}\right) \int \hat{\chi}_{i j}\left(\mathbf{r}, \mathbf{r}^{\prime}, \omega\right) d \mathbf{r}^{\prime} \\
& =\int \hat{\chi}_{i k}\left(\mathbf{r}, \mathbf{r}^{\prime}, \omega\right) \nabla_{k}^{\prime} \nabla_{j}^{\prime} V_{0}\left(\mathbf{r}^{\prime}\right) d \mathbf{r}^{\prime}+\frac{\omega^{2}}{c} n_{0}(\mathbf{r}) \delta_{i j},
\end{aligned}
$$

where

$$
V_{0}(\mathbf{r})=\sum_{k} v_{0}\left(\mathbf{r}-\mathbf{R}_{k}\right)
$$

$v_{0}(\mathbf{r})$ is the bare potential of one impurity centered at origin, $n_{0}(\mathbf{r})$ is the ground-state electron density, and $\omega_{p}=\sqrt{4 \pi \bar{n}_{0}}$ is plasma frequency of the homogeneous electron gas without impurities. Applying Eq. (2) twice with respect to the integration over $\mathbf{r}$ and $\mathbf{r}^{\prime}$ in Eq. (1), and using the expression for the density-response function

$$
\chi\left(\mathbf{r}, \mathbf{r}^{\prime}, \omega\right)=-\frac{c}{\omega^{2}} \nabla_{i} \cdot \hat{\chi}_{i j}\left(\mathbf{r}, \mathbf{r}^{\prime}, \omega\right) \cdot \nabla_{j}^{\prime},
$$

together with the static sum rule [36]

$$
\int \chi\left(\mathbf{r}, \mathbf{r}^{\prime}, 0\right) \nabla_{i}^{\prime} V_{0}\left(\mathbf{r}^{\prime}\right) d \mathbf{r}^{\prime}=\nabla_{i} n_{0}(\mathbf{r}),
$$

we eventually write the current density as

$$
\begin{aligned}
j_{i}(\omega)= & \frac{\omega}{i\left(\omega^{2}-\omega_{p}^{2}\right)}\left(\bar{n}_{0} E_{\mathrm{ext}, i}+\frac{1}{\left(\omega^{2}-\omega_{p}^{2}\right) V}\right. \\
& \times\left\{\int _ { V } d \mathbf { r } d \mathbf { r } ^ { \prime } [ \nabla _ { i } V _ { 0 } ( \mathbf { r } ) ] \left[\chi\left(\mathbf{r}, \mathbf{r}^{\prime}, \omega\right)\right.\right. \\
& \left.\left.\left.-\chi\left(\mathbf{r}, \mathbf{r}^{\prime}, 0\right)\right]\left[\nabla_{j}^{\prime} V_{0}\left(\mathbf{r}^{\prime}\right)\right]\right\} E_{\mathrm{ext}, j}\right) .
\end{aligned}
$$

In order to calculate the frequency-dependent complex resistivity tensor $\rho_{i j}(\omega)$ defined as

$$
E_{i}(\omega)=-\rho_{i j}(\omega) j_{j}(\omega),
$$

we need to know the total electric field $\mathbf{E}$, which is the sum of the external electric field $\mathbf{E}_{\text {ext }}$ and the field that is induced by the current itself. From Maxwell's equations, and since $\nabla \times \mathbf{B}$ averages out to zero, one has

$$
\mathbf{E}(\omega)=\mathbf{E}_{\mathrm{ext}}(\omega)+\frac{4 \pi i}{\omega} \mathbf{j}(\omega) .
$$

Equation (7) substituted into Eq. (5) implicitly determines the resistivity tensor $\rho_{i j}(\omega)$ in the general case of finite frequency. As we show below, in the specific case of $\omega \rightarrow 0$, this relation becomes explicit. Notice that both $\mathbf{E}$ and $\mathbf{j}$ vanish in the $\omega \rightarrow 0$ limit. The external electric field, on the other hand, is clearly finite and given by $\mathbf{E}_{\mathrm{ext}}=-\frac{4 \pi i}{\omega} \mathbf{j}$ in the same $\omega \rightarrow 0$ limit.

Substituting this expression for $\mathbf{E}_{\mathrm{ext}}$ in the second term on the right-hand side of Eq. (5), putting $\mathbf{j}=\frac{\omega}{4 \pi i}\left(\mathbf{E}-\mathbf{E}_{\text {ext }}\right)$ on the left-hand side, and lastly, noting that

$$
\chi\left(\mathbf{r}, \mathbf{r}^{\prime}, \omega\right)-\left.\chi\left(\mathbf{r}, \mathbf{r}^{\prime}, 0\right) \simeq i \omega \frac{\partial \operatorname{Im} \chi\left(\mathbf{r}, \mathbf{r}^{\prime}, \omega\right)}{\partial \omega}\right|_{\omega=0},
$$

we arrive at our main formal result:

$$
\begin{aligned}
\rho_{i j}= & -\frac{1}{\bar{n}_{0}^{2} V} \int_{V}\left[\nabla_{i} V_{0}(\mathbf{r})\right]\left[\nabla_{j}^{\prime} V_{0}\left(\mathbf{r}^{\prime}\right)\right] \\
& \times\left.\frac{\partial \operatorname{Im} \chi\left(\mathbf{r}, \mathbf{r}^{\prime}, \omega\right)}{\partial \omega}\right|_{\omega=0} d \mathbf{r} d \mathbf{r}^{\prime}
\end{aligned}
$$

Equation (9) is the formal solution to the problem of the resistivity in terms of the density-density response function $\chi$ of the interacting inhomogeneous electron gas with impurities. We now substitute the operator equality

$$
\left.\frac{\partial \chi(\omega)}{\partial \omega}\right|_{\omega=0}=-\left.\chi(0) \frac{\partial \chi^{-1}(\omega)}{\partial \omega}\right|_{\omega=0} \chi(0)
$$

into Eq. (9), and apply again the static sum rule of Eq. (4). Recalling that [18]

$$
\chi^{-1}\left(\mathbf{r}, \mathbf{r}^{\prime}, \omega\right)=\chi_{s}^{-1}\left(\mathbf{r}, \mathbf{r}^{\prime}, \omega\right)-f_{x c}\left(\mathbf{r}, \mathbf{r}^{\prime}, \omega\right)-\frac{1}{\left|\mathbf{r}-\mathbf{r}^{\prime}\right|},
$$

where $\chi_{s}$ is the "Kohn-Sham" density-density response function of the noninteracting system with ground-state density $n_{0}(\mathbf{r})$ and $f_{x c}$ is the dynamical exchange and correlation kernel, we conveniently rewrite Eq. (9) in terms of $\chi_{s}$ and the $f_{x c}$ as follows:

$$
\rho_{i j}=\rho_{1, i j}+\rho_{2, i j},
$$

$$
\begin{aligned}
\rho_{1, i j}= & -\frac{1}{\bar{n}_{0}^{2} V} \int_{V}\left[\nabla_{i} V_{s}(\mathbf{r})\right]\left[\nabla_{j}^{\prime} V_{s}\left(\mathbf{r}^{\prime}\right)\right] \\
& \times\left.\frac{\partial \operatorname{Im} \chi_{s}\left(\mathbf{r}, \mathbf{r}^{\prime}, \omega\right)}{\partial \omega}\right|_{\omega=0} d \mathbf{r} d \mathbf{r}^{\prime},
\end{aligned}
$$

where $V_{s}(\mathbf{r})$ is the static Kohn-Sham (KS) potential,

$$
\begin{aligned}
\rho_{2, i j}= & -\frac{1}{\bar{n}_{0}^{2} V} \int_{V}\left[\nabla_{i} n_{0}(\mathbf{r})\right]\left[\nabla_{j}^{\prime} n_{0}\left(\mathbf{r}^{\prime}\right)\right] \\
& \times\left.\frac{\partial \operatorname{Im} f_{x c}\left(\mathbf{r}, \mathbf{r}^{\prime}, \omega\right)}{\partial \omega}\right|_{\omega=0} d \mathbf{r} d \mathbf{r}^{\prime} .
\end{aligned}
$$


Notice that in Eq. (12) we have used the "Kohn-Sham version" of the static sum rule $\int \chi_{s}\left(\mathbf{r}, \mathbf{r}^{\prime}, 0\right) \nabla_{i}^{\prime} V_{s}\left(\mathbf{r}^{\prime}\right) d \mathbf{r}^{\prime}=\nabla_{i} n_{0}(\mathbf{r})$ to introduce the Kohn-Sham potential $V_{s}$.

Equation (12) is the single-particle (KS) contribution to the resistivity. Under the assumption that the electrons feel only one impurity at a time, (i.e., the coherent scattering of an electron from more than one impurity is neglected), we replace the full KS potential $V_{s}(\mathbf{r})$ by the KS potential associated with a single impurity in the electron gas, which we further assume to be spherically symmetric, i.e., $V_{s}(\mathbf{r})=V_{s}(r)$. The KS response function $\chi_{s}\left(\mathbf{r}, \mathbf{r}^{\prime}, \omega\right)$ is computed accordingly. The normalization volume is taken to be equal to the volume per impurity, i.e., $V=1 / n_{i}$. It can be proved without further assumptions $[33,36]$ that Eq. (12), thus modified, yields exactly

$$
\rho_{1}=\frac{k_{F} n_{i}}{\bar{n}_{0}} \sigma_{t r}\left(k_{F}\right),
$$

where $\sigma_{t r}$ is the transport cross section, which can be calculated by means of the standard $T$-matrix (phase-shift) technique in the potential $V_{s}(\mathbf{r})[12,13]$.

Equation (13) is the dynamical exchange-correlation contribution. If the frequency dependence of $f_{x c}$ is neglectedas one does, for example, in the adiabatic approximation to TDDFT-then $f_{x c}$ is purely real and Eq. (13) yields $\rho_{2}=0$. This result, combined with the discussion of the previous paragraph, leads to the important conclusion that the adiabatic approximation to TDDFT is equivalent to the classical potential-scattering ( $T$-matrix) approach as far as the calculation of the resistivity is concerned.

To find the many-body contribution to the resistivity from Eq. (13), we need a good approximation to the dynamical exchange and correlation kernel $f_{x c}$. It is known [38] that $f_{x c}\left(\mathbf{r}, \mathbf{r}^{\prime}\right)$ is strongly nonlocal (i.e., a long-ranged function of $\left.\left|\mathbf{r}-\mathbf{r}^{\prime}\right|\right)$ and this nonlocality is crucial to a proper description of many-body effects in transport phenomena, even on a qualitative level [32]. This immediately poses the problem of construction of a reasonably accurate nonlocal approximation for $f_{x c}$. In a recent paper [32] we have shown how this can be done starting from an exact representation of the scalar $f_{x c}$ kernel in terms of the tensor exchange-correlation kernel $\hat{f}_{x c}$ of time-dependent current density functional theory. This representation reads

$$
\begin{aligned}
f_{x c}= & -\frac{\omega^{2}}{c} \nabla^{-2} \nabla \cdot\left\{\hat{f}_{x c}+\left(\hat{\chi}_{s}^{-1}-\hat{f}_{x c}\right)\left[\hat{T}\left(\hat{\chi}_{s}^{-1}-\hat{f}_{x c}\right) \hat{T}\right]^{-1}\right. \\
& \left.\times\left(\hat{\chi}_{s}^{-1}-\hat{f}_{x c}\right)-\hat{\chi}_{s}^{-1}\left(\hat{T} \hat{\chi}_{s}^{-1} \hat{T}\right)^{-1} \hat{\chi}_{s}^{-1}\right\} \cdot \nabla \nabla^{-2},
\end{aligned}
$$

where $\hat{\chi}_{s}$ is the KS current-current response function, $\hat{T}$ is the projector operator onto the subspace of transverse vector fields (i.e., divergence-free fields), and $\nabla^{-2}$ is the inverse of the Laplace operator: $\nabla^{-2} f(\mathbf{r})=-\int d \mathbf{r}^{\prime} \frac{f\left(\mathbf{r}^{\prime}\right)}{4 \pi\left|\mathbf{r}-\mathbf{r}^{\prime}\right|}$ in three dimensions [39].

By making use of the local density approximation (LDA) for the tensor $\hat{f}_{x c}$ [20] in the right-hand side of Eq. (15), we obtain a nonlocal approximation for the scalar $f_{x c}$, which satisfies the static zero-force sum-rule requirements [32,38], and can, therefore, be considered a promisingly accurate approximation for transport problems. At the same time, in Ref. [40], the LDA to the exchange and correlation kernel $\hat{f}_{x c}$ of the TDCDFT has been worked out within the framework of the hydrodynamics of inhomogeneous viscous electron liquid. A simplified form for this kernel (neglecting the bulk viscosity and keeping only the shear viscosity) is [40]

$$
\begin{aligned}
i \omega \hat{f}_{x c, i j}= & \sum_{j} \frac{1}{n_{0}} \nabla_{j} \tilde{\eta}_{x c}(\omega) \nabla_{j} \frac{1}{n_{0}}+\frac{1}{n_{0}} \nabla_{j} \tilde{\eta}_{x c}(\omega) \nabla_{i} \frac{1}{n_{0}} \\
& -\frac{2}{d} \frac{1}{n_{0}} \nabla_{i} \tilde{\eta}_{x c}(\omega) \nabla_{j} \frac{1}{n_{0}}
\end{aligned}
$$

where $\tilde{\eta}_{x c}(\omega)=\eta_{x c}(\omega)-\frac{\mu_{x c}(\omega)}{i \omega}$ is the complex viscoelastic coefficient of the homogeneous electron liquid, with $\eta_{x c}$ being the xc shear viscosity and $\mu_{x c}$ the xc shear modulus $\left(\eta_{x c}\right.$ and $\mu_{x c}$ are both real; $d$ is the number of space dimensions).

We therefore use Eq. (13) with $f_{x c}$ given by Eq. (15) and $\hat{f}_{x c}$ given by Eq. (16) with the viscoelastic constants of the homogeneous electron liquid evaluated at the local density $n_{0}(\mathbf{r})$. The values of these constants are taken from Ref. [28]. Notice that $\hat{f}_{x c}$ has a real part controlled by the shear modulus $\mu_{x c}$ and an imaginary part controlled by the shear viscosity $\eta_{x c}$. The perturbative treatment suggested in Ref. [29] led to the conclusion that the dynamical correction to the resistivity would be controlled exclusively by the shear viscosity. However, we see that this is not the case in the present treatment. Because the Kohn-Sham response function $\chi_{s}$ has both a real and an imaginary part, the imaginary part of $f_{x c}$, as given by Eq. (15), is now controlled by both $\mu_{x c}$ and $\eta_{x c}$.

In Fig. 1, we present results for resistivity for substitutional impurities of atomic number $Z$ from 11 through 32 in an aluminum host. The latter is modeled as a jellium with WignerSeitz radius $r_{s}=2.07$. The single-particle contribution $\rho_{1}$ calculated from Eq. (14) and represented by the dashed chained curve (black) is found to be in agreement with earlier calculations [12,13]. The total resistivity, including dynamical exchange and correlation contributions, is represented by the

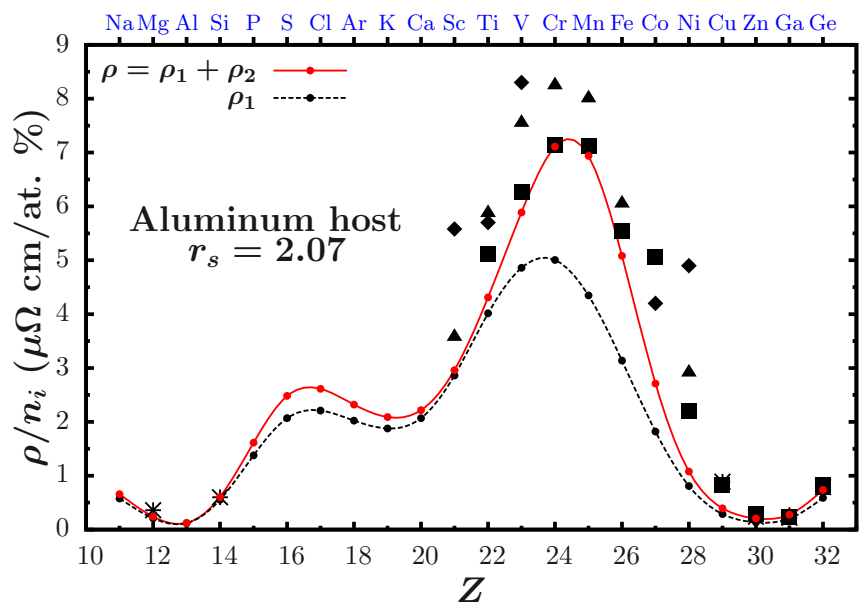

FIG. 1. (Color online) Residual resistivity of aluminum due to substitutional impurities of atomic number $Z$, as a function of $Z$. The solid chained curve (red online) is our result with inclusion of the dynamical exchange and correlations [the sum of $\rho_{1}$ and $\rho_{2}$ obtained with use of Eqs. (14) and (13), respectively]. The dashed chained curve (black) is the result of the single-particle theory ( $\rho_{1}$ only). Solid symbols are experimental data compiled from Ref. [37]. 
solid chained curve (red). An improved agreement between theory and experiment can be clearly seen from the figure. The dynamical corrections are sizable and indeed of the right order of magnitude to account for the observed discrepancy between available theoretical calculations and experimental data.

Further analysis shows that the dominant contribution to the dynamical corrections does not come from the viscosity, $\eta_{x c}$, but from the shear modulus, $\mu_{x c}$, contribution to the xc kernel of Eq. (16). In fact, the contribution from $\eta_{x c}$ is one to two orders of magnitude smaller than that from $\mu_{x c}$. This is apparently in contrast with the discussion of Ref. [29], in which the dynamical correction was expressed solely in terms of $\eta_{x c}$.

Physically, however, the emergence of $\mu_{x c}$ as the main contributor to the resistivity makes physical sense. The homogeneous kernel of Eq. (16) is "collisionless," i.e., does not include the effect of electron-impurity collisions on the viscosity and the shear modulus. Mathematically, these quantities were obtained from a "high-frequency" calculation [28] in which the frequency remains higher than the electronimpurity collision rate. Therefore, we expect that the true zero-frequency limit of the viscosity, which should ultimately control the excess dissipation and hence the dynamical correction to the resistivity, should be quite different from the collisionless $\eta_{x c}$ used in Eq. (16). In fact, according to a dispersion relation argument put forth in Ref. [30], a reasonable expression for the zero-frequency limit of the viscosity (i.e., the true collisional viscosity) is $\eta_{x c}(0)=\mu_{x c} \tau$, where $\mu_{x c}$ is the high-frequency shear modulus, and $1 / \tau$ is the electron-impurity scattering rate. The same conclusion was reached in Ref. [28] [see, in particular, Eq. (5.28)] where the collisional (i.e., $\omega=0$ ) viscosity was found to be equal to the collisionless shear modulus multiplied by the collision time. It now appears that the xc kernel constructed via Eq. (15) does take into account the effect of electron-impurity collisions. It does so via the imaginary part of the noninteracting response function $\chi_{s}$ which, indeed, is calculated in the presence of the impurity. It is the imaginary part of $\chi_{s}$ that, combined with the real part of $\hat{f}_{x c}$ (i.e., $\mu_{x c}$ ), generates the main contribution to the dynamical resistivity. We interpret this fact as an indication that Eq. (15) is dressing the homogeneous kernel (16) by including the effect of electron-impurity collisions.

In conclusion, we have developed the nonadiabatic timedependent density functional formalism for a systematic calculation of the $\mathrm{dc}$ residual resistivity of metals with impurities. The contribution to the resistivity arising from the many-body interactions has been expressed through the dynamical exchange and correlation kernel $f_{x c}$. We have shown that all the dynamical effects of the electron-electron interaction are contained in the frequency dependence of $f_{x c}$. The adiabatic approximation, which neglects this frequency dependence, is exactly equivalent to the conventional singleparticle potential-scattering theory of the resistivity, provided the coherent scattering from multiple impurities is neglected as well. Our representation of the single-particle and the manybody contributions to the resistivity through the Kohn-Sham response function and the dynamical xc kernel, respectively, is fit to use in band-structure calculations [41], and can provide guidance in calculating the resistance of nanoscale conductors.

V.U.N. acknowledges support from National Science Council, Taiwan, Grant No. 100-2112-M-001-025-MY3. G.V. acknowledges support from DOE Grant No. DEFG0205ER46203.
[1] N. F. Mott and H. Jones, The Theory of the Properties of Metals and Alloys (Dover, New-York, 1958).

[2] R. Landauer, IBM J. Res. Dev. 1, 223 (1957).

[3] R. Landauer, J. Phys.: Condens. Matter 1, 8099 (1989).

[4] M. Büttiker, Y. Imry, R. Landauer, and S. Pinhas, Phys. Rev. B 31, 6207 (1985).

[5] M. Büttiker, Phys. Rev. Lett. 57, 1761 (1986).

[6] S. Datta, Electronic Transport in Mesoscopic Systems (Cambridge University Press, New York, 1997).

[7] M. D. Ventra, Electrical Transport in Nanoscale Systems (Cambridge University Press, Cambridge, 2008).

[8] G. Stefanucci and R. van Leeuwen, Nonequilibrium ManyBody Theory of Quantum Systems: A Modern Introduction (Cambridge University Press, Cambridge, 2013).

[9] Y. Meir and N. S. Wingreen, Phys. Rev. Lett. 68, 2512 (1992).

[10] G. Vignale, in Fundamentals of Time-Dependent Density Functional Theory, edited by M. A. L. Marques, N. T. Maitra, F. M. S. Nogueira, E. K. U. Gross, and A. Rubio (Springer, Berlin, 2012), p. 457.

[11] C. A. Ullrich, Time-Dependent Density-Functional Theory: Concepts and Applications (Oxford University Press, Oxford, 2012).

[12] R. M. Nieminen and M. Puska, J. Phys. F: Met. Phys. 10, L123 (1980).
[13] M. J. Puska and R. M. Nieminen, Phys. Rev. B 27, 6121 (1983).

[14] P. Hohenberg and W. Kohn, Phys. Rev. 136, B864 (1964).

[15] W. Kohn and L. J. Sham, Phys. Rev. 140, A1133 (1965).

[16] J. P. Perdew, R. G. Parr, M. Levy, and J. L. Balduz, Phys. Rev. Lett. 49, 1691 (1982).

[17] C. Toher, A. Filippetti, S. Sanvito, and K. Burke, Phys. Rev. Lett. 95, 146402 (2005).

[18] E. K. U. Gross and W. Kohn, Phys. Rev. Lett. 55, 2850 (1985).

[19] S. K. Ghosh and A. K. Dhara, Phys. Rev. A 38, 1149 (1988).

[20] G. Vignale and W. Kohn, Phys. Rev. Lett. 77, 2037 (1996).

[21] S. Stefanucci and C.-O. Almbladh, Europhys. Lett. 67, 14 (2004).

[22] N. Sai, M. Zwolak, G. Vignale, and M. Di Ventra, Phys. Rev. Lett. 94, 186810 (2005).

[23] J. Jung, P. Bokes, and R. W. Godby, Phys. Rev. Lett. 98, 259701 (2007).

[24] N. Sai, M. Zwolak, G. Vignale, and M. Di Ventra, Phys. Rev. Lett. 98, 259702 (2007).

[25] M. Koentopp, K. Burke, and F. Evers, Phys. Rev. B 73, 121403(R) (2006).

[26] P. Bokes, J. Jung, and R. W. Godby, Phys. Rev. B 76, 125433 (2007).

[27] S. Conti, R. Nifosì, and M. Tosi, J. Phys.: Condens. Matter 9, L475 (1997). 
[28] S. Conti and G. Vignale, Phys. Rev. B 60, 7966 (1999).

[29] G. Vignale and M. Di Ventra, Phys. Rev. B 79, 014201 (2009).

[30] D. Roy, G. Vignale, and M. Di Ventra, Phys. Rev. B 83, 075428 (2011).

[31] S. Kurth and G. Stefanucci, Phys. Rev. Lett. 111, 030601 (2013).

[32] V. U. Nazarov, J. M. Pitarke, Y. Takada, G. Vignale, and Y.-C. Chang, Phys. Rev. B 76, 205103 (2007).

[33] V. U. Nazarov, J. M. Pitarke, Y. Takada, G. Vignale, and Y.-C. Chang, Int. J. Mod. Phys. B 22, 3813 (2008).

[34] In Eq. (1), the integration over $\mathbf{r}^{\prime}$ gives the microscopic current density by virtue of the Kubo formula, while the integration over $\mathbf{r}$ with the division by $V$ represents the averaging in the system uniform on the macroscopic scale. All many-body and possible quantum interference effects are contained in the current-density response function $\hat{\chi}$.

[35] Equation (2) is a generalization for infinite systems of the corresponding sum rule of Ref. [42] accurately taking into account the potential of the uniform positive background, which as well as the bare potential of the impurities $V_{0}(\mathbf{r})$, is external to the electron subsystem.
[36] V. U. Nazarov, J. M. Pitarke, C. S. Kim, and Y. Takada, Phys. Rev. B 71, 121106(R) (2005).

[37] E. Babic, Z. Vucic, I. Zoric, M. Ocko, B. Leontic, E. Girt, and R. Krsnik, Solid State Commun. 10, 691 (1972); G. Boato, M. Bugo, and C. Rizzuto, Nuovo Cimento 45, 226 (1966); Y. Fukai, Phys. Rev. 186, 697 (1969).

[38] G. Vignale, Phys. Lett. A 209, 206 (1995).

[39] In the case of the one-dimensional inhomogeneity, Eq. (15) simplifies to an expression which does not involve $\hat{\chi}_{s}[42,43]$. This, however, is not true in the general case.

[40] G. Vignale, C. A. Ullrich, and S. Conti, Phys. Rev. Lett. 79, 4878 (1997).

[41] N. Papanikolaou, R. Zeller, P. H. Dederichs, and N. Stefanou, Comput. Mater. Sci. 8, 131 (1997).

[42] G. Vignale and W. Kohn, in Electronic Density Functional Theory: Recent Progress and New Directions, edited by J. Dobson, M. P. Das, and G. Vignale (Plenum, New York, 1998).

[43] M. Dion and K. Burke, Phys. Rev. A 72, 020502(R) (2005). 